\title{
Brain Surgery
}

National Cancer Institute

\section{Source}

National Cancer Institute. Brain Surgery. NCI Thesaurus. Code C154268.

Surgery performed on any part of the brain. 